\title{
RADAR Commentary: Evolution and Current Status of Dosimetry in Nuclear Medicine
}

\author{
Michael G. Stabin ${ }^{1}$, Robert M. Sharkey ${ }^{2}$, and Jeffry A. Siegel ${ }^{3}$ \\ ${ }^{1}$ Department of Radiology and Radiological Sciences, Vanderbilt University, Nashville, Tennessee; ${ }^{2}$ Center for Molecular Medicine \\ and Immunology, Garden State Cancer Center, Belleville, New Jersey; and ${ }^{3}$ Nuclear Physics Enterprises, Marlton, New Jersey
}

$\mathbf{T}$ here is a long history of absorbed dose calculations being performed to evaluate the risks involved in the application of radiopharmaceuticals to medical studies. In the 1940s, Marinelli, Quimby, and Hine (1-3) published a set of articles that presented a summary and approach to radionuclide dosimetry. In 1956 Loevinger et al. (4) summarized the field at that point, and then in 1968 the newly formed MIRD Committee of the Society of Nuclear Medicine published the original MIRD schema as MIRD Pamphlet No. 1 (5). In 2003, the RAdiation Dose Assessment Resource (RADAR) task group of the Society of Nuclear Medicine demonstrated an easily understood and unified method for calculating internal doses in nuclear medicine or radiation protection and implemented the method in easy-to-use Web and computer tools $(6)$. Over these many years, numerous articles have been written describing new and improved dosimetry models and methods, better quantification schemes for counting, scintillation camera imaging, and radiobiologic modeling, along with recognition that baseline pretherapy dose-limiting organ radiosensitivity needed to be part of any predictive treatment plan.

Internal dosimetry has always been an integral part of radionuclide drug development, being used primarily from 2 perspectives. Most often, dosimetry is performed as an $a$ posteriori analysis after radiopharmaceutical administration (diagnostic and therapeutic) simply to provide standard dose estimates for that procedure. According to U.S. Food and Drug Administration requirements, all phase I clinical trials involving a radioactive component must include studies that will obtain sufficient data for these "characterizational" dosimetry calculations, which are done for all source and target regions in a limited number of subjects. The second type of dosimetry is used to guide therapy before treatment and is therefore performed as an a priori or predictive analysis. In external-beam or brachytherapy, dosimetry is evaluated for all patients but generally only

\footnotetext{
Received Feb. 2, 2011; revision accepted Mar. 16, 2011.

For correspondence or reprints contact: Michael G. Stabin, Department of Radiology and Radiological Sciences, Vanderbilt University, 116121 st Ave. S., Nashville, TN 37232-2675.

E-mail: michael.g.stabin@vanderbilt.edu

COPYRIGHT @ 2011 by the Society of Nuclear Medicine, Inc.

DOI: $10.2967 /$ jnumed.111.088666
}

for a single region corresponding to the treatment area. Unfortunately, unlike the universal agreement on the importance and need for characterizational dosimetry in drug development, dosimetry generally is not used to guide radionuclide therapy, despite the substantial efforts to develop and standardize internal dose methods. There is currently no consensus within the scientific community on the utility of predictive dosimetry, and regulatory agencies do not require its use for determining a treatment activity prescription, because they believe there is little evidence indicating that dosimetry is predictive (of either toxicity or response), as compared with simpler administration methods (administered activity or activity adjusted for body mass or surface area). The only exception is the case of ${ }^{131}$ I-tositumomab (Bexxar; GlaxoSmithKline) (7), for which a relatively simple yet useful (whole-body) dosimetry approach is used to provide a patient-individualized activity administration.

Radiation has the unique distinction among other forms of therapy (e.g., drugs or biologics) that it can be detected external to the patient, allowing organ and body activity and clearance estimates from which individualized radiation doses can be determined. Thus, dosimetry has the potential to become an important and clinically useful scientific tool, because it offers a unique opportunity to develop an appropriate treatment activity prescription on a per-patient basis and to quantify the risk involved in the administration of a therapeutic agent to tumors and normal tissues in each patient before the treatment is given, as is always done for external-beam radiotherapy. In contrast, this is not done in chemotherapy, for which administered quantities are empirically determined. Building a consensus to support predictive dosimetry will occur only if there are data clearly showing its benefit in treatment planning and if evidence to support this position is available.

\section{CURRENT STATUS}

Radionuclide treatment of thyroid cancer and hyperthyroidism with $\mathrm{Na}^{131} \mathrm{I}$ has the longest history in nuclear medicine. Fixed-activity treatments generally have been used, but recently studies have shown that in ${ }^{131} \mathrm{I}$ in thyroid therapy, better outcomes are indeed seen when a radiation dose-based criterion is used to guide therapy $(8,9)$; furthermore, use of an activity-based criterion has been shown to 
result in administering too much activity to most patients (10). During the early development of radioimmunotherapy, predictive dosimetry was used primarily for treatments designed to give myeloablative doses of the radiolabeled antibodies, with hematopoietic function restored via bone marrow transplantation $(11,12)$. Predictive dosimetry in myeloablative settings has been extremely useful; however, there are not many trials with radioactive agents in this setting, and this type of dosimetry requires that a careful quantitative imaging analysis be performed for the second organ of toxicity. At least one nonmyeloablative trial started with a pretherapy dosimetry study to estimate the red marrow dose (first using a blood-based method and then an image-based method) and then fixed the therapeutic activity level at the amount needed to deliver a fixed absorbed dose to the marrow (13). However, the Food and Drug Administration required these protocols to be changed so that the treatment activity was administered solely on the basis of activity per unit body surface area, citing the lack of credible evidence that dosimetry for radiolabeled antibodies could be related to experience with ${ }^{131}$ I-thyroid therapy. Today most nonmyeloablative radionuclide therapy clinical trials continue to use a fixed-activity treatment prescription, with the notable exception of ${ }^{131}$ I-tositumomab, for which the administered activity is adjusted according to each patient's biokinetics and body weight or surface area. In the use of ${ }^{131}$ I-tositumomab, because of highly variable clearance rates between patients, whole-body dosimetry estimated from a pretherapy study is used to prescribe the therapeutic amount of activity to administer on a patientspecific basis $(7,14)$, with the idea of controlling toxicity, at acceptable response levels, by limiting dose to marrow (with whole-body dose as a surrogate for marrow dose). More recently, peptide radionuclide receptor therapy has offered an opportunity for the dosimetry community to develop models that can predict not only the potential for nephrotoxicity but also responses for individual patients. A pretherapy dosimetry and imaging study is needed to define the individual's dosing regimen on the basis of biodistribution, and factors that affect a patient's sensitivity to the radiation exposure may be used to make further patientspecific adjustments. There is a dichotomy of purpose for any pretherapy study. If appropriate regions of interest are drawn, such images can be analyzed to predict toxicity (e.g., using the whole body or bone marrow regions) or response (e.g., tumors). At present, pretherapy studies, if done at all, are generally performed to enable toxicity prediction because the ability to predict response has proven to be much more challenging, particularly when many tumors are present. The balance between accuracy and clinical feasibility has yet to be established but is necessary to create practical and clinically meaningful predictive dosimetry models.

With radiolabeled antibody (IgG) trials unanimously finding hematologic toxicity to be dose-limiting, identifying pretherapy factors that could predict toxicity should have great interest. Some factors are intuitive, such as that when there is bone marrow involvement, this would most likely result in a greater deposition of radioactivity within the bone marrow, enhancing the exposure of critical stem cells. Another intuitive factor that must be considered is the number of prior therapies with chemotherapeutic agents that can cause a compromised bone marrow reserve. There is no universally accepted model for predicting marrow toxicity accurately in individual patients on a pretherapy basis, nor is there a clear understanding of how to use this information to adjust the treatment activity prescription (i.e., directly, by modification of the calculational schema using parameters that are different for patients with and without pretherapy factors, or indirectly, by stratifying patients into clinically distinct subpopulations). Perhaps the best way to derive a therapeutic dose would be to estimate a patient-specific absorbed, or biologically effective, dose determined from an appropriate pretherapy assessment and then to adjust by a factor, representative of pretherapy bone marrow radiosensitivity, that would make this dose predictive of the likelihood and degree of myelosuppression. The risk of severe hematologic toxicity in patients treated with Bexxar and ibritumomab tiuxetan (Zevalin; Spectrum Pharmaceuticals, Inc.) has been minimized by using relatively simple stratification, that is, by limiting treatment to patients with less than $25 \%$ of their bone marrow involved with disease and a predetermined platelet count. Baechler et al. (15) argued against the use of dosimetry in patients treated with either Bexxar or Zevalin, suggesting instead the use of time from last chemotherapy treatment as most indicative of the likelihood of myelotoxicity for non-Hodgkin lymphoma patients; however, they assumed incorrectly that bone marrow dose was essentially constant for these patients.

Red marrow absorbed dose can be derived by determining the amount of activity in blood samples taken over time or by quantification via imaging. Total body dose has been shown to be as good as red marrow dose in certain cases depending on radionuclide and total body-to-blood biokinetics (16). Therefore, it is important to appreciate that model differences may be justifiable in certain cases and that not all findings should be lumped together. Imaging methods provide a more direct link to individualize the assessment of bone marrow uptake, and they were found to be helpful when diffuse bone marrow involvement was present (16). Shen et al. (17), using a ${ }^{90}$ Y-antibody in radioimmunotherapy in a patient population without a significant impact of marrow involvement and previous myelosuppressive chemotherapies, obtained a correlation coefficient of 0.85 between marrow dose and observed marrow toxicity, using patient-specific marrow mass estimated from CT images. However, this method may not be as successful for pretreated patient populations or subjects with extensive marrow disease, as is the case for non-Hodgkin lymphoma patients. Importantly, patients who are recovering from chemotherapy may have hyperproliferating bone marrow, which may result in marrow that is more radiosensitive 
and capable of being visualized on imaging. Nevertheless, bone marrow involvement is usually assessed by biopsy, not imaging, and is subject to sampling error (18). Bone marrow involvement is not an issue restricted to lymphoma; patients with medullary thyroid cancer often have unappreciated spread in the marrow, as shown by Mirallie et al. (19). Indeed, radioimmunotherapy trials in this indication using an ${ }^{131}$ I-labeled hapten-peptide pretargeted with an anti-carcinoembryonic antigen antibody have noted these patients to be more susceptible to myelotoxicity, and there is even some suggestion that survival improvements may be related to elimination of disseminated tumor in the marrow $(20,21)$. Adopting a policy of assessing bone marrow involvement from an imaging study with the treatment agent might have allowed for better stratification, but the status of the marrow reserve and its radiosensitivity would still not be accounted for. In 2003, a retrospective analysis of serum FLT3-L levels (a biologic marker that was indicative of bone marrow status) found that absorbed dose estimates corrected for FLT3-L levels correlated well with hematologic toxicity (22). This was the first indication that a biologic marker had the potential to be used in concert with marrow radiation absorbed dose to improve toxicity predictions. There have been no other clinical studies to examine the utility of this or other bone marrow markers in an effort to complete our understanding of how to pair dosimetry with biologic markers for better treatment planning. Further hampering efforts to improve dose-toxicity correlations, absorbed doses are generally correlated with peripheral blood count indicators of toxicity (using percentage decrease, grade, or nadir), which are not strictly linearly related to response except at low nadir levels (e.g., platelet nadirs of 45,000 and 90,000 observed in 2 patients do not correspond to a factor-of- 2 difference in outcome for these patients) (16). When patients were categorized by the severity of their bone marrow toxicity, those patients experiencing grade $0-2$ platelet toxicity had a significantly reduced dose-toxicity correlation compared with those exhibiting grade 3 or 4 toxicity (22). Further, normal peripheral blood cell counts are typically considered sufficient indicators of patient tolerance for additional myelosuppressive treatment; however, these counts do not reliably predict patient response to further radionuclide therapy. This has been shown to be true with FLT3-L in subjects with solid tumors (22).

A goal of dosimetry-guided radiotherapy is to deliver the highest possible dose to tumor while managing dose to normal tissues, whether in a myeloablative or nonmyeloablative setting. To optimize such therapy, should the approach be based on dose-limiting toxicity or tumor control? A model has been proposed to address both toxicity and response (23), but it has had limited application and generally the treatment approach is to prescribe a therapeutic activity that will limit toxicity and, it is hoped, result in a meaningful tumor dose for an adequate response. Pauwels et al. found a convincing relationship between tumor dose and response in neuroendocrine tumors in their study of 22 patients with ${ }^{90}$ Y-DOTATOC $\left({ }^{90} \mathrm{Y}\right.$-OctreoTher [Novartis AG], an antisomatostatin peptide) (24), using PET with ${ }^{86} \mathrm{Y}$ to characterize the in vivo kinetics of the agent. Others, however (e.g., Sgouros et al. (25) and Baechler et al. (15)), have not been able to establish strong relationships, but this may be due to the patient populations studied. Sharkey et al. also reported no obvious relationship between response and tumor targeting in lymphoma patients, noting responses in some lesions that were not visualized even by external scintigraphy (26). Koral et al. (27) have found good tumor dose-response correlation in previously untreated patient populations as opposed to the results of Sharkey et al., which were obtained in patients with disease relapse. The effects of prior treatment are expected to confound the dose-response relationship, and it is important to stress that care must be exercised when trying to characterize or combine the results obtained in various clinical trials, because the generally enormous variations in tumors and patients limit our ability to derive meaningful models. Better focused and more uniform data are needed (28). In lymphoma, it is important to keep in mind that the unconjugated antibodies used in these treatments have antitumor effects, and therefore responses would not necessarily occur only as a result of radiation exposure $(29,30)$.

Peptides have also become important radionuclide targeting agents, but unlike antibodies, these small molecules clear quickly from the blood, reducing the risk of hematologic toxicity. Most radioactivity is cleared from the body by urinary excretion, and therefore radiation dose to the kidneys and the urinary bladder becomes the primary concern. Nephrotoxicity is particularly problematic because these effects are not immediately apparent; the full severity of the condition may not be known for 6 mo or more. This time line creates a problem in designing clinical trials, because typical phase I dose escalation studies would need to be delayed for many months before all effects were fully known. Thus, these treatments would benefit greatly from reliable predictive dosimetry. Initial studies with a ${ }^{90} \mathrm{Y}-$ labeled antisomatostatin receptor-binding peptide resorted first to using characterizational dosimetry to define renal uptake. Escalation of the administered activity proceeded in these early trials, but there were a few instances of serious late renal toxicities (31-33). In addition to these studies, another clinical trial investigating a ${ }^{166} \mathrm{Ho}-b o n e-$ targeting agent discovered end-stage renal toxicity when exceptionally high levels of radioactivity were administered to achieve marrow ablation $(34,35)$. In that situation, imaging studies with ${ }^{166} \mathrm{Ho}$ were problematic and quantification of renal uptake was difficult. It became apparent that the high dose rates experienced by the subjects' kidneys during early renal clearance needed to be considered in relating calculated radiation dose to the observed effects, although interpretation was complicated by preexisting renal damage in some patients.

These experiences highlighted the need to improve predictive dosimetry for radionuclides that clear primarily 
through the kidneys. Several groups involved in the testing of radiolabeled peptides provided important insights indicating that the prediction of late renal toxicity could be improved if, first, the actual mass of the kidneys was derived from anatomic images; second, the activity uptake in the kidney was considered to be nonuniformly distributed, with most of the radioactivity being retained in the cortex; third, a linear quadratic biologic response model was used to estimate the biologic effective dose; and finally, if patient preexisting conditions were considered $(36,37)$. These findings represent important advancements for the use of radiolabeled peptides in the future, and the MIRD Committee's Pamphlet No. 20 (38) has stated that this approach leads to the best currently available predictive model to minimize nephrotoxicity in peptide radionuclide receptor therapy. Unfortunately, this MIRD pamphlet was issued with methodologic errors, questionable assumptions, and an incomplete consideration of different candidate radiobiologic models (39). Several issues still need to be addressed before we can claim that a truly useful predictive dosimetry model exists, namely the accuracy of kidney activity quantification from medical images; the accuracy of the use of single-cycle dose estimates to predict the total kidney dose in a multicycle treatment; the use of fixed radiobiologic parameters in the linear quadratic model, which are not mathematically meaningful in application to individual patients; and the application of corrections (e.g., by a patient stratification paradigm or direct adjustment of biologic model parameters) for preexisting conditions that can greatly affect the response of the kidneys to radiation.

\section{CONCLUDING REMARKS}

The single most important issue arguing for patientspecific dosimetry is how to optimize the therapeutic window for each patient, ensuring the tumor receives the highest possible dose, while minimizing radiation dose and toxicity to normal tissues. Establishing reliable dose-response or dose-toxicity correlations is an important first step, and prospective clinical trials are needed to demonstrate that individualized treatment activity prescriptions can be designed and implemented appropriately. No therapeutic agent has truly advanced to this position, because not only are there not enough data, but it has also proven difficult to predict the precise amount of any agent that then will give a known response for individual patients while maintaining manageable toxicity levels (except in the case of radionuclide therapy performed in a myeloablative setting). The maximum amount of activity tolerated or the maximum tolerated dose of any radionuclide treatment is generally determined during a phase I clinical trial, and the hope is that the treatment activity level at the maximum tolerated dose would be the best possible in every patient. However, usually a substantial number of patients experience considerably less toxicity (e.g., $<$ grade 2) than allowed at the treatment maximum tolerated dose, and any further adjustments most often are made to ensure that the population exceeding the dose-limiting toxicity is minimized rather than to optimize the number of patients being treated at the maximum toxicity allowed (16). Thus, many radionuclide therapy patients will experience low toxicity and may benefit from a higher treatment activity prescription. This is the challenge for dosimetryto guide radionuclide therapy; that is, can it provide a reliable approach to optimize the number of patients treated at the maximum toxicity level and also minimize the number of patients experiencing more severe toxicity (16)? It must also be demonstrated that those patients experiencing, for example, a toxicity of no more than grade 2 are less responsive to treatment and would likely benefit more if they were given a higher activity prescription to result in perhaps grade 3 or 4 toxicity. Therefore, data are still needed to establish methods for using dosimetry, radiobiologic modeling, and pretherapy dose-limiting organ status to adjust therapy activity prescriptions on an individual basis to optimize efficacy while avoiding severe toxicity.

In the short term, dosimetry will continue to be a descriptive tool used in treatment modalities that rely on therapeutic radionuclides. In previously untreated patient populations, as is the case in thyroid cancer treatment with $\mathrm{Na}^{131}$ I or targeted radiotherapy used as a frontline therapy (40), dosimetry on its own may play an important role if the aim is to limit toxicity or optimize response, although in the latter case dosimetry may need to be coupled with consideration of treatment-related factors, such as antibody effects, known to influence tumor response. In the heavily pretreated patient populations generally receiving radionuclide therapy or in patient populations with certain preexisting risk factors, it is almost certain that absorbed dose calculated using standardized models, or even dose coupled to a radiobiologic model, would require modification by factors, or biologic marker results, indicative of pretherapy dose-limiting organ status or consideration of factors that may affect tumor response, such as varying radiosensitivity. Perhaps, an equally effective treatment strategy would result if an appropriate patient stratification algorithm were used, whether or not a predictive dosimetry analysis is performed as well (e.g., that used for non-Hodgkin lymphoma patients treated with Bexxar or Zevalin, respectively). The most appropriate models will most certainly vary, depending on their applicability for a particular radiolabeled agent used for treatment in a given patient population.

Internal dosimetry professionals and the radionuclide therapy community are not taking a sufficiently proactive role in coupling absorbed dose or biologic effective dose with these other important patient-specific pretherapy factors and appear to be unappreciative that vast differences are to be expected for different radionuclide-agent constructs in different patient populations. Changing the role of dosimetry to become more directly involved in planning of patients' therapy will require improvement in our understanding of dose-response relationships. This improvement 
of course cannot happen if no absorbed dose, or biologic effective dose, estimates are calculated and will be hampered if they are calculated without using well-established methodologies and appropriate radiobiologic models and corrections.

Given adherence to high-quality dosimetry and understanding of patient characteristics, several questions need to be answered. What scale of dosimetry is needed for understanding and predicting radiation effects (e.g., whole organ, suborgan regions, voxel regions, single cells)? Can a meaningful dosimetric approach be based on an easily measurable macroscopic spatial scale so that it could be reliably implemented on a routine clinical basis? What are the optimal schedules for treatment for a given therapeutic agent? Fractionation might reduce normal-tissue toxicity, but might it also reduce the already low dose-rate exposure within the tumor, thus reducing the therapeutic potential? Biokinetics established in one pretherapy dosimetry study may not be predictive of what will occur in subsequent treatments. Kobe et al. (8) did not rely only on the biologic clearance rates from their pretherapy studies but studied the clearance rates during therapy as well and adjusted administered activity as needed to meet a dose target. How do factors such as linear energy transfer, dose rate, biodistribution, and particle range affect tumor and healthy-tissue responses to radiation? The answers to these and other important questions likely will vary depending on radionuclide treatment and patient population and be well answered only with a strong partnership between physicians and physicists who are used to dealing with individualized dose-response characterization. Notably, in Europe there is a legal imperative to perform dose estimates in planning of radionuclide therapy, and those who have heeded this imperative have demonstrated improved patient outcomes with reduced institutional costs $(8)$ and the ability to predict tumor response (24), renal toxicity $(36,37,39)$, and myelotoxicity (22). Partnerships between investigators in the United States and colleagues in Europe, and a commitment to build an experience base with different radiopharmaceuticals in different patient populations, could significantly advance the field of dosimetry and ultimately result in better care for patients (41) - the foremost goal of all such efforts. It is important to begin learning how best to alter the planned therapy activity prescription on the basis of absorbed dose, radiobiologic modeling, and pretherapy factors such as baseline organ status, previous therapies, and individual radiosensitivity. The goal for any predictive model is to estimate in an individual patient the response and potential for dose-limiting toxicity and to use this information in adjusting the individual's treatment plan. Further, any such model must be clinically feasible to implement-a significant challenge. Minimally, the model should at least make better use of factors known to be predictive of toxicity or response, such as patient pretherapy status and cold antibody effects, to modify absorbed dose values. Without such an approach, progress in understanding radiation dose-effect relationships is hindered, causing many patients to receive suboptimal therapy (thus reducing response rates) or to experience unanticipated toxicity and, in the case of thyroid diseases, significant under- or overdosing resulting in unnecessary exposures to their families and others (10) and higher costs for the institution (8). More work needs to be done by the dosimetry community to prove that dosimetry is not only worthwhile but also required to optimally guide radionuclide therapy. Many investigators have shown predictive dosimetry to be extremely useful in a research setting for a wide variety of radionuclide therapies, but these methods need to be more thoroughly tested before they are considered to represent accurate procedures that can be implemented on a routine clinical basis. The goal of optimally guided radionuclide therapy has not yet been realized, but progress has been good in recent years and continued study should be encouraged.

\section{DISCLOSURE STATEMENT}

The costs of publication of this article were defrayed in part by the payment of page charges. Therefore, and solely to indicate this fact, this article is hereby marked "advertisement" in accordance with 18 USC section 1734.

\section{REFERENCES}

1. Marinelli L, Quimby E, Hine G. Dosage determination with radioactive isotopes: practical considerations in therapy and protection. Am J Roentgenol Radium Ther. 1948;59:260-281.

2. Quimby E, Feitelberg S. Radioactive Isotopes in Medicine and Biology. Philadelphia, PA: Lea and Febiger; 1963.

3. Hine G, Brownell G. Radiation Dosimetry. New York, NY: Academic Press; 1956.

4. Loevinger R, Japha E, Brownell G. Discrete radioisotope processes. In: Hine G, Brownell G, eds. Radiation Dosimetry. New York, NY: Academic Press; 1956:694-802.

5. Loevinger R, Berman M. MIRD Pamphlet No. 1, Revised: A Revised Schema for Calculating the Absorbed Dose from Biologically Distributed Radionuclides. New York, NY: Society of Nuclear Medicine; 1976.

6. Stabin MG, Siegel JA. Physical models and dose factors for use in internal dose assessment. Health Phys. 2003;85:294-310.

7. Siegel JA, Kroll S, Regan D, Kaminski MS, Wahl RL. A practical methodology for patient release after tositumomab and ${ }^{131}$ I-tositumomab therapy. J Nucl Med. 2002;43:354-363.

8. Kobe C, Eschner W, Sudbrock F, et al. Graves' disease and radioiodine therapy: is success of ablation dependent on the achieved dose above 200 Gy? Nuklearmedizin. 2008;47:13-17.

9. Flux GD, Haq M, Chittenden SJ, et al. A dose-effect correlation for radioiodine ablation in differentiated thyroid cancer. Eur J Nucl Med Mol Imaging. 2010;37:270-275.

10. Jonsson H, Mattsson S. Excess radiation absorbed doses from non-optimized radioiodine treatment of hyperthyroidism. Radiat Prot Dosimetry. 2004;108:107-114.

11. Press OW, Eary JF, Appelbaum FR, et al. Radiolabeled-antibody therapy of B-cell lymphoma with autologous bone marrow support. N Engl J Med. 1993; 329:1219-1224.

12. Press OW, Eary JF, Badger CC, et al. Treatment of refractory non-Hodgkin's lymphoma with radiolabeled MB-1 (anti-CD37) antibody. J Clin Oncol. 1989; 7:1027-1038.

13. Juweid ME, Sharkey RM, Behr T, et al. Radioimmunotherapy of patients with small-volume tumors using iodine-131-labeled anti-CEA monoclonal antibody NP-4 F(ab') $)_{2}$. J Nucl Med. 1996;37:1504-1510.

14. Wahl RL, Zasadny KR, MacFarlane D, et al. Iodine-131 anti-B1 antibody for Bcell lymphoma: an update on the Michigan phase I experience. J Nucl Med. 1998;39(suppl)21S-27S.

15. Baechler S, Hobbs RF, Jacene HA, Bochud FO, Wahl RL, Sgouros GS. Predicting hematologic toxicity in patients undergoing radioimmunotherapy with ${ }^{90} \mathrm{Y}$ ibritumomab tiuxetan or ${ }^{131}$ I-tositumomab. J Nucl Med. 2010;51:1878-1884. 
16. Siegel JA. Establishing a clinically meaningful predictive model of hematologic toxicity in nonmyeloablative targeted radiotherapy: practical aspects and limitations of red marrow dosimetry. Cancer Biother Radiopharm. 2005;20:126-140.

17. Shen S, Meredith RF, Duan J, et al. Improved prediction of myelotoxicity using a patient-specific imaging dose estimate for non-marrow-targeting ${ }^{90} \mathrm{Y}$-antibody therapy. J Nucl Med. 2002;43:1245-1253.

18. Sgouros G. Bone marrow dosimetry for radioimmunotherapy: theoretical considerations. J Nucl Med. 1993;34:689-694.

19. Mirallie E, Vuillez JP, Bardet S, et al. High frequency of bone/bone marrow involvement in advanced medullary thyroid cancer. J Clin Endocrinol Metab. 2005;90:779-788.

20. Chatal JF, Campion L, Kraeber-Bodere F, et al. Survival improvement in patients with medullary thyroid carcinoma who undergo pretargeted anti-carcinoembryonicantigen radioimmunotherapy: a collaborative study with the French endocrine tumor group. J Clin Oncol. 2006;24:1705-1711.

21. Kraeber-Bodere F, Goldenberg DM, Chatal JF, Barbet J. Pretargeted radioimmunotherapy in the treatment of metastatic medullary thyroid cancer. Curr Oncol. 2009;16:3-8.

22. Siegel JA, Yeldell D, Goldenberg DM, et al. Red marrow radiation dose adjustment using plasma FLT3-L cytokine levels: improved correlations between hematologic toxicity and bone marrow dose for radioimmunotherapy patients. $\mathrm{J} \mathrm{Nucl}$ Med. 2003;44:67-76.

23. Shen S, Duan J, Meredith RF. Model prediction of treatment planning for dosefractionated radioimmunotherapy. Cancer. 2002;94:1264-1269.

24. Pauwels S, Barone R, Walrand S, et al. Practical dosimetry of peptide receptor radionuclide therapy with ${ }^{90}$ Y-labeled somatostatin analogs. J Nucl Med. 2005;46(suppl):92S-98S.

25. Sgouros G, Squeri S, Ballangrud AM, et al. Patient-specific, 3-dimensional dosimetry in non-Hodgkin's lymphoma patients treated with ${ }^{131} \mathrm{I}$-anti-B1 antibody: assessment of tumor dose-response. J Nucl Med. 2003;44:260-268.

26. Sharkey RM, Brenner A, Burton J, et al. Radioimmunotherapy of non-Hodgkin's lymphoma with ${ }^{90}$ Y-DOTA humanized anti-CD22 IgG ( ${ }^{90}$ Y-epratuzumab): do tumor targeting and dosimetry predict therapeutic response? J Nucl Med. 2003;44:2000-2018.

27. Koral KF, Kaminski MS, Wahl RL. Correlation of tumor radiation-absorbed dose with response is easier to find in previously untreated patients. $J$ Nucl Med. 2003; $44: 1541-1543$.

28. DeNardo SJ, Williams LF, Leigh BR, Wahl RL. Choosing an optimal radioimmunotherapy dose for clinical response. Cancer. 2002;94:1275-1286.
29. Cardarelli PM, Quinn M, Buckman D, et al. Binding to CD20 by anti-B1 antibody or $\mathrm{F}\left(\mathrm{ab}^{\prime}\right)_{2}$ is sufficient for induction of apoptosis in B-cell lines. Cancer Immunol Immunother. 2002;51:15-24.

30. Hernandez MC, Knox SJ. Radiobiology of radioimmunotherapy: targeting CD20 B-cell antigen in non-Hodgkin's lymphoma. Int J Radiat Oncol Biol Phys. 2004;59:1274-1287.

31. Moll S, Nickeleit V, Mueller-Brand J, Brunner FP, Maecke HR, Mihatsch MJ. A new cause of renal thrombotic microangiopathy: yttrium 90-DOTATOC internal radiotherapy. Am J Kidney Dis. 2001;37:847-851.

32. Cybulla M, Weiner SM, Otte A. End-stage renal disease after treatment with ${ }^{90} \mathrm{Y}-$ DOTATOC. Eur J Nucl Med. 2001;28:1552-1554.

33. Behr TM, Béhé M, Kluge G, et al. Nephrotoxicity versus anti-tumour efficacy in radiopeptide therapy: facts and myths about the Scylla and Charybdis. Eur J Nucl Med Mol Imaging. 2002;29:277-279.

34. Breitz H, Wendt R, Stabin M, Bouchet L, Wessels B. Dosimetry of high dose skeletal targeted radiotherapy (STR) with ${ }^{166}$ Ho-DOTMP. Cancer Biother Radiopharm. 2003; 18:225-230.

35. Breitz HB, Wendt RE III, Stabin MS, et al. ${ }^{166}$ Ho-DOTMP radiationabsorbed dose estimation for skeletal targeted radiotherapy. J Nucl Med. 2006;47:534-542.

36. Barone R, Borson-Chazot F, Valkema R, et al. Patient-specific dosimetry in predicting renal toxicity with ${ }^{90}$ Y-DOTATOC: relevance of kidney volume and dose rate in finding a dose-effect relationship. J Nucl Med. 2005;46(suppl):99S$106 \mathrm{~S}$.

37. Bodei L, Cremonesi M, Ferrari M, et al. Long-term evaluation of renal toxicity after peptide receptor radionuclide therapy with ${ }^{90}$ Y-DOTATOC and ${ }^{177} \mathrm{Lu}-$ DOTATATE: the role of associated risk factors. Eur J Nucl Med Mol Imaging. 2008;35:1847-1856.

38. Wessels BW, Konijnenberg MW, Dale RG, et al. MIRD Pamphlet No. 20: the effect of model assumptions on kidney dosimetry and response-implications for radionuclide therapy. J Nucl Med. 2008;49:1884-1899.

39. Siegel JA, Stabin MG, Sharkey RM. Renal dosimetry in peptide radionuclide receptor therapy. Cancer Biother Radiopharm. 2010;25:581-588.

40. Kaminski MS, Estes J, Tuck M, et al. Iodine I 131 tositumomab therapy for previously untreated follicular lymphoma (FL) [abstract]. Proc Am Soc Clin Oncol. 2000;19(suppl):5A.

41. Stabin MG. The case for patient-specific dosimetry in radionuclide therapy. Cancer Biother Radiopharm. 2008;23:273-284. 